\title{
Identification of the HSP70-II gene in Leishmania braziliensis HSP70 locus: genomic organization and UTRs characterization
}

\author{
César A Ramírez ${ }^{1,2}$, José M Requena² and Concepción J Puerta ${ }^{1 *}$
}

\begin{abstract}
Background: The heat stress suffered by Leishmania sp during its digenetic life-cycle is a key trigger for its stage differentiation. In Leishmania subgenera two classes of HSP70 genes differing in their 3' UTR were described. Although the presence of HSP70-I genes was previously suggested in Leishmania (Viannia) braziliensis, HSP70-II genes had been reluctant to be uncovered.
\end{abstract}

Results: Here, we report the existence of two types of HSP7O genes in L. braziliensis and the genomic organization of the HSP70 locus. RT-PCR experiments were used to map the untranslated regions (UTR) of both types of genes. The 3' UTR-II has a low sequence identity (55-57\%) when compared with this region in other Leishmania species. In contrast, the $5^{\prime}$ UTR, common to both types of genes, and the $3^{\prime}$ UTR-I were found to be highly conserved among all Leishmania species (77-81\%). Southern blot assays suggested that L. braziliensis HSP70 gene cluster may contain around 6 tandemly-repeated HSP70-I genes followed by one HSP70-II gene, located at chromosome 28. Northern blot analysis indicated that levels of both types of mRNAs are not affected by heat shock.

Conclusions: This study has led to establishing the composition and structure of the HSP70 locus of L. braziliensis, complementing the information available in the GeneDB genome database for this species. L. braziliensis HSP70 gene regulation does not seem to operate by mRNA stabilization as occurs in other Leishmania species.

\section{Background}

The Leishmania genus involves about 20 species that infect humans, causing different clinical manifestations ranging from self-healing cutaneous lesions (CL), mucosal lesions (MCL) to fatal visceral infections (VL) [1]. More than 350 million people are considered at risk of contracting leishmaniases, and some 2 million new cases occur yearly [2]. In Latin America, CL and MCL are neglected public health problems endemic in 22 countries. Many species of the subgenus Viannia cause the majority of CL cases but MCL is principally caused by Leishmania (Viannia) braziliensis [3]. Canine leishmaniases, caused either by Leishmania infantum or by $L$. braziliensis, is also widespread in South America, being

\footnotetext{
* Correspondence: cpuerta@javeriana.edu.co

'Laboratorio de Parasitología Molecular, Departamento de Microbiología, Facultad de Ciencias, Pontificia Universidad Javeriana, Carrera 7 No. 43-82, Edificio 52, Oficina 608, Bogotá, Colombia

Full list of author information is available at the end of the article
}

among the most important canine vector-borne diseases occurring in this region [4].

During its digenetic life cycle, the Leishmania parasite needs to adapt from the environmental (vector) temperature to the mammalian-host temperature $\left(37^{\circ} \mathrm{C}\right)$. As a result, the heat shock response is induced and the heat shock proteins (HSPs) are expected to play important roles in the adaptation process, influencing the developmental change from promastigotes in sandflies to amastigotes in mammalian hosts [5-10]. Among HSPs, HSP70 is the most highly conserved in both sequence and function. Proteins of the HSP70 family are central components of many fundamental cellular processes, including the folding and assembly of newly synthesized proteins, refolding of misfolded and aggregated proteins, membrane translocation of organellar and secretory proteins, proteolytic degradation of unstable proteins, and control of regulatory protein activity [11-14].
C Biomed Central 
Two classes of HSP70 genes, HSP70-I and HSP70-II, sharing the 5' untranslated region (UTR) and the coding region but differing in their 3' UTR, have been described in several Leishmania species like L. infantum, Leishmania major, Leishmania tropica, Leishmania mexicana and Leishmania amazonensis $[15,16]$. In general, these genes are arranged in a single genomic cluster that contains five or six HSP70-I copies, followed by one HSP70II copy. In L. infantum, it has been demonstrated that whereas HSP70-I mRNAs accumulate in response to heat shock treatment, and are translated at both 26 and $37^{\circ} \mathrm{C}$, HSP70-II mRNAs do not show a temperaturedependent accumulation, but show preferential translation at heat shock temperatures [15].

Given that Leishmania genes are transcribed into polycistronic RNA precursors that need to be further processed into individual mRNAs by trans-splicing and polyadenylation, post-transcriptional regulation represents the main level of controlling gene expression in these parasites [17]. Currently, multiple efforts are being dedicated to identify cis- and trans-elements involved in the modulation of mRNA processing, mRNA stabilization/destabilization, mRNA half-life, or translation efficiency. Although regulatory sequences have been identified in both 5' and 3' UTRs, most of them have been located in the 3' UTRs [18-23]. For instance, preferential translation of HSP83 in Leishmania requires a thermosensitive polypyrimidine-rich element (PPT) in the 3' UTR [24].

Our knowledge on the organization and expression of HSP70 genes in Leishmania species of the subgenus Viannia is scanty. Although, the sequence for the $L$. braziliensis genome has recently been published [25], unfortunately, the genomic sequence found in the GeneDB database presents several gaps that hinder to elucidate the organization of the HSP7O locus in $L$. braziliensis. Moreover, in a preliminary work, it was documented by hybridization assays the existence of HSP70-I genes in L. braziliensis, but evidence on the presence of HSP70-II genes was not obtained [16]. In this work, we have determined the 5' and 3' UTRs for the HSP70 L. braziliensis genes, demonstrating the existence of the HSP70-II gene in this Viannia species, and established the organization of the HSP7O locus. Also the expression of both types of HSP7O genes was assessed.

\section{Methods}

\section{Parasite cultures}

Promastigotes of L. braziliensis (MHOM/BR/75/M2904) were cultured in vitro at $26^{\circ} \mathrm{C}$ in Schneiders 's insect medium (Sigma Aldrich, Inc., St. Louis, USA) supplemented with $20 \%$ heat-inactivated fetal calf serum (Eurobio, Inc., Les Ulis, France), and $0.1 \mu \mathrm{g} / \mathrm{mL}$ of 6 -
Biopterin (Sigma Aldrich, Inc., St. Louis, USA). For heat shock treatments, $10 \mathrm{~mL}$-aliquots of L. braziliensis promastigote cultures at logarithmic phase $\left(5-9 \times 10^{6}\right.$ promastigotes $\mathrm{mL}^{-1}$ ) were transferred into $50 \mathrm{~mL}$ flasks, and incubated at $32^{\circ} \mathrm{C}, 35^{\circ} \mathrm{C}$ or $37^{\circ} \mathrm{C}$ for two hours. Afterwards, parasites were harvested for DNA or RNA extraction.

\section{Southern and Northern blot analyses}

Total DNA from L. braziliensis cells was isolated using the phenol-chloroform-isoamilic alcohol method [26]. Two $\mu$ g of DNA were digested with several restriction enzymes according to the manufacturer specifications (Promega, Inc., Madison, WI, USA), electrophoresed on 0.8\% low electroendosmosis agarose gels (Conda Pronadisa, Inc., Madrid, Spain), and transferred to nylon membrane (Roche, Inc., Mannheim Germany) by standard methods [26]. Total RNA from promastigotes was isolated using the Total Quick RNA Cells and Tissues (TALENT, Inc., Trieste, Italy). Four $\mu$ g per line were separated on $1.5 \%(\mathrm{w} / \mathrm{v})$ low electroendosmosis agarose/ MOPS/formaldehyde gels and transferred to nylon membranes. L. braziliensis chromosomes were prepared from promastigotes, harvested during log phase, washed and embedded in $0.6 \%$ low melting agarose (GIBCO BRL, Inc., N.Y, USA) plugs, which were finally soaked in a lysis solution (0.5 M EDTA, pH 9; $1 \% \mathrm{SDS}, 1 \mathrm{mg} / \mathrm{mL}$ proteinase $\mathrm{K})$ at $50^{\circ} \mathrm{C}$ during $48 \mathrm{~h}$.

After three washes with $0.2 \mathrm{M}$ EDTA for $2 \mathrm{~h}$ each one, the blocks were loaded directly into the wells of $1 \%$ agarose NA gel (Amersham Bioscience, Inc., Uppsala, Sweden), sealed in place, separated by pulsed homogeneous electric field gel electrophoresis (PFGE) using a Pharmacia Biotech Gene Navigator apparatus at $100 \mathrm{~V}$, $120^{\circ}$ separation angle and switch times varying from 100 $\mathrm{s} / 7 \mathrm{~h} ; 200 \mathrm{~s} / 10 \mathrm{~h}$ and $500 \mathrm{~s} / 20 \mathrm{~h}$, and transferred to nylon membranes. The following probes were used: 3' UTR-I (clone pTLb3HSP70-D, SmaI/EcoRI digested), 3' UTR-II (clone pTLb3H70-11B, HincII/EcoRI digested), and intercoding (IR-HSP70, clone pLbHSP70-IR-E, PstI digested). They were labeled with digoxigenin by randomly primed synthesis using the DIG High Prime DNA Labeling kit (Roche, Inc., Mannheim, Germany). Hybridizations and immunological detection were performed using the Detection Starter kit II (Roche, Inc., Mannheim, Germany) according to manufacturer's instructions. Finally, membranes were exposed on Curix RP2 plus medical X-Ray film (AGFA, Mortsel, Belgium).

\section{Cloning UTR sequences and in silico analyses}

First-strand cDNA synthesis was carried out from $L$. braziliensis total RNA using an oligo-dT primer and a cDNA synthesis kit (LKB Pharmacia, New Jersey, USA). Amplification of the UTRs was performed from poly-T 
primed-cDNA using specific primers: LbSL (5'CGCTA TATAAGTATCAGTTTC3') and Lb181 (5'TGCAACC CGATCATGACCAAG 3') for the 5' UTR, and Lb1824 (5'GATCATGACCAAGATGTACCAGAG 3') and Poly T EcoRI (5'CGGAATTCTTTTTTTTTTTTTTTTTTT 3') for the 3' UTR-I (see Additional file 1). Briefly, $20 \mu \mathrm{L}$ reactions containing $2 \mu \mathrm{L}$ of cDNA, $1 \mathrm{X}$ reaction buffer (10 mM Tris- $\mathrm{HCl} \mathrm{pH} 9.0,50 \mathrm{mM} \mathrm{KCl}, 0.1 \%$ Triton $\mathrm{X}$ 100), $1.5 \mathrm{mM} \mathrm{MgCl}_{2}, 0.7 \mathrm{mM}$ of dNTP mix, $0.2 \mu \mathrm{M}$ of each primer, $2 \mathrm{M}$ betain, and 0.06 units per $\mu \mathrm{L}$ of expand high fidelity enzyme (Roche, Branford, USA) were prepared. For 3' UTR-II amplification, $1 \mathrm{mM}$ $\mathrm{MgCl}_{2}$ and $0.5 \mu \mathrm{M}$ of each primer were used. An MJ Research PTC-100 DNA thermocycler was used for the reaction with the following amplification profile: $95^{\circ} \mathrm{C} / 5$ min (initial denaturation), 35 cycles at $92^{\circ} \mathrm{C} / 0.5 \mathrm{~min}, 58^{\circ}$ $\mathrm{C} / 0.5 \mathrm{~min}$ and $72^{\circ} \mathrm{C} / 1 \mathrm{~min}$, with a final incubation at $72^{\circ} \mathrm{C}$ for $10 \mathrm{~min}$. All the amplified fragments were resolved in agarose gels and visualized under UV exposure after ethidium bromide staining. RT-PCR products were excised from gels, purified using GFX Gel Band Purification kit (Amersham Biosciences, GE Healthcare, Chalfont St. Giles, Buckinghamshire, England) and cloned in the pGEM $^{\mathbb{R}}$-T Easy plasmid (Promega, Madison, WI, USA); pCR ${ }^{\circledR}$ II (Invitrogen, California, USA) or pCR2.1 (Invitrogen, California, USA) plasmids. The following clones were obtained: pLbHSP70-5B containing the 5' UTR, pTLb3HSP70-D for the 3' UTR-I, pLb3H70-11B for the 3' UTR-II, and pLbHSP70-IR-E for the intercoding region. The sequences were determined using the Big Dye Terminators v3.1 kit (Applied Biosystem, California, USA) by automatic sequencing at the Servicio de Genómica (Parque Científico de Madrid, Universidad Autónoma de Madrid). In order to deduce the HSP70 mRNA UTRs from other Leishmania species LALIGN (http://www.ch.embnet.org/software/LALIGN_form.html) analyzes were performed. A ClustalW analysis (http://www.ebi.ac.uk/Tools/clustalw2/index.html) for multiple alignments of sequences, were carried out to determine the similarity among them.

\section{Results and discussion}

The L. braziliensis HSP70 locus contains two types of HSP70 genes

Gene structure and expression of the HSP70 protein have been well characterized in $L$. infantum $[7,15,16,27]$ and other trypanosomatids of medical importance as L. major [28], Trypanosoma brucei [29,30], and Trypanosoma cruzi [31-36]; nonetheless, little is known about their genes in L. braziliensis. A previous study showed the presence of the HSP70-I genes in this parasite; however, it was not determined their copy number. These authors also reported that using the 3' UTR-II region from L. infantum as probe, it was not possible to detect the presence of HSP70-II genes in L. braziliensis [16].

In order to determine the genomic organization of HSP70 genes in L. braziliensis, we searched in the $L$. braziliensis genome database at GeneDB for entries containing HSP70 sequences. One complete HSP70 gene (GeneDB ID: LbrM28_v2.2990) and two incomplete sequences, LbrM28_v2.2980 (bearing a 5'-fragment of the gene encoding the N-terminal region of HSP70) and LbrM28_v2.2970 (encoding the C-terminal protein region) were found (Figure 1). Between both fragments of HSP7O genes, a sequence gap of undetermined length is annotated in the database. Even though LbrM28_v2.2980 and LbrM28_v2.2970 might be part of the same gene, the sequenced regions do not overlap. All three sequences are tandemly organized on the minus strand of chromosome 28. No other entries for HSP70 genes were found in the L. braziliensis database. This genomic organization of the HSP7O genes in $L$. braziliensis resembled that found in other Leishmania species of the subgenus Leishmania, i.e. a locus containing tandemly repeated HSP7O genes [16]. However,

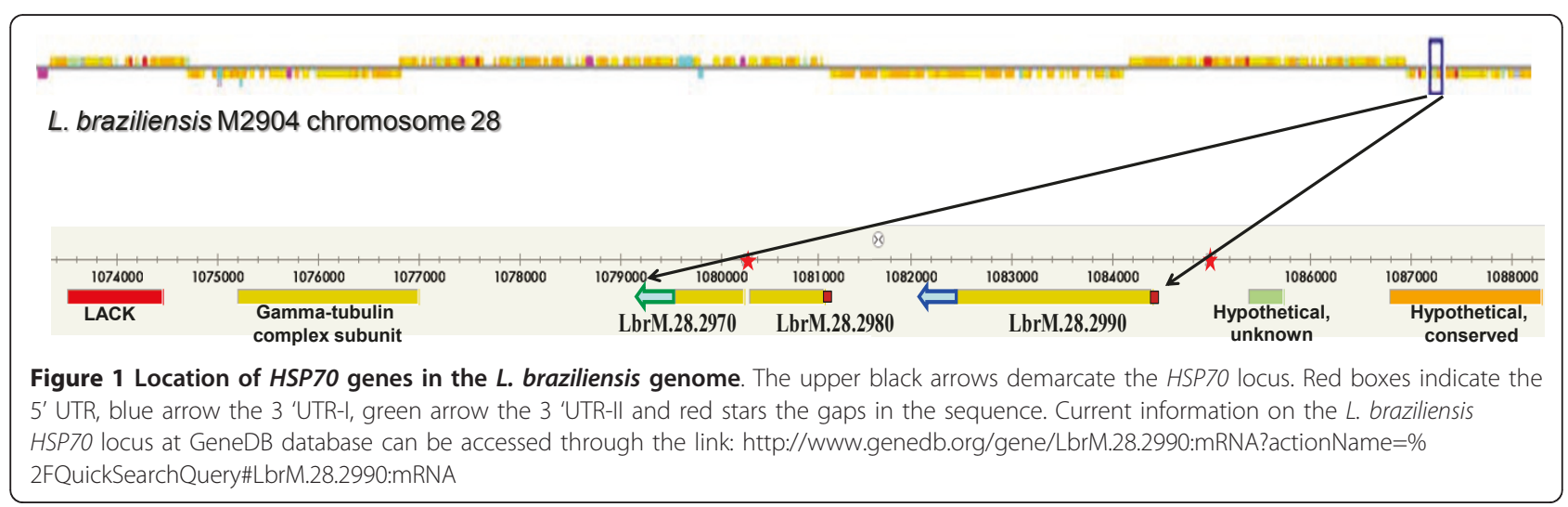


tandem gene arrays are among the most challenging to resolve correctly using current genome sequencing technology, since repetitive sequence reads tend to collapse into a single contig when no variation exists to distinguish them. Thus, in order to determine the copy number of HSP7O genes composing the locus, and possible sequence divergence existing between the different genes, we designed specific oligonucleotides to cloning the complete intercoding regions (UTRs+intergenic regions), from genomic DNA, and also the 5'- and 3'UTRs by RT-PCR from poly-A ${ }^{+}$RNA (see Methods section for further details). A single amplification fragment for the intercoding region was obtained and, after cloning, its sequence was found to be conserved (99.8\% of sequence identity) with the sequence located between genes LbrM28_v2.2990 and LbrM28_v2.2980. These findings suggest a high conservation of intercoding regions in the HSP70 locus; however, a more accurate determination of degree of conservation would require the sequencing of several additional clones.

On the other hand, using RT-PCR and specific oligonucleotides, it was possible to determine the 5' UTR sequence, which is $198 \mathrm{bp}$ in length (Figure 2). This sequence has been deposited in the GenBank database under the accession number JF449363. In spite of the high sequence identity, among the four sequences analyzed, two microsatellite length polymorphisms in the 5'-UTR were observed (see Additional file 2), suggesting the existence of allelic polymorphisms in the L. braziliensis HSP70 locus. In addition, the alignment of the 5' UTR with the genomic sequences allowed us to deduce

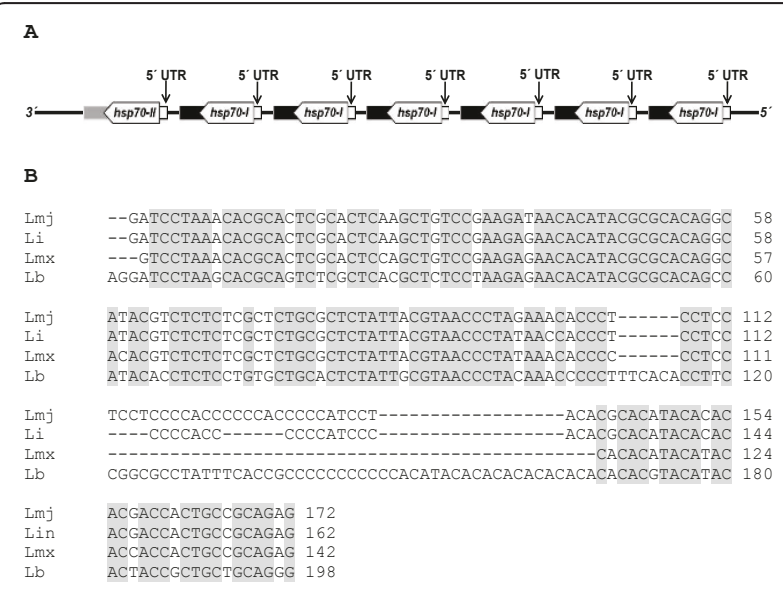

Figure 2 Position and sequence of the $5^{\prime}$ UTR of HSP70 cluster from L. braziliensis. (A). Graphical representation of the $5^{\prime}$ UTR position in the HSP7O cluster of L. braziliensis. (B). Multiple sequence alignment among HSP70 5' UTR from L. braziliensis (Lb: JF449363), L. major (Lmj: LmjF28.2770), L. infantum (Li: LinJ28_V3.2960) and L. mexicana (Lmx: LmxM28.2770). Conserved sequences are shaded in gray. that the AG splicing acceptor is located 194 and 199 nucleotides upstream of the start codon of LbrM28_v2.2990 and LbrM28_v2.2980 genes, respectively. Also, in both entries, 28 nucleotides upstream of the splicing acceptor site, an identical polypyrimidine tract of 22 nucleotides in length (5' CTCCCCTTTCTCT CTCTGCCCC 3') with a U/C ratio of 0.62 is observed. It is likely that this polypyrimidine tract is relevant for the trans-splicing process. Furthermore, in the coding regions contained in clones pLbHSP70-IR-E and pLbHSP70-5B, two transitions of guanine by adenine were found. The first one causes a change of cysteine by tyrosine, and the other produces a change of glutamic acid by lysine in the encoded polypeptides (Additional file 2). This variability, together with the microheterogenicity detected in the 5' UTR, supports the existence of at least four HSP70 genes in the diploid genome of $L$. braziliensis; but this resulted to be an underestimation as we demonstrate below.

Regarding the 3' UTR of HSP70 genes, two types of sequences were cloned and sequenced (GenBank JF449364 and JF449365), showing a high sequence divergence each other. After sequence comparison, they could be assigned to the two types of 3' UTR (I and II) described in HSP7O genes of other Leishmania species (Figures 3 and 4). Thus, we determined that the 3' UTR type I is 936 nucleotides long, and would correspond to the LbrM28_v2.2990 entry (and possibly other genes of the HSP70 gene cluster, see below), and the 3' UTR type II is 932 nucleotides long and would be associated with LbrM28_v2.2970 entry. The 3' UTR-II was found 100\% identical with the genomic sequence located downstream of LbrM28_v2.2970, whereas the 3' UTR-I had 99.1\% of sequence identity with the sequence located downstream of LbrM28_v2.2990 entry, suggesting that the cloned 3' UTR-I might correspond to other HSP70 gene in the cluster (see below). According to the 3' UTR-I sequence, the polyadenylation in the LbrM28_v2.2990 transcript would occur after two adenines, and $187 \mathrm{bp}$ upstream of the putative polypyrimidine tract previously mentioned. Regarding the LbrM28_v2.2970 gene, the polyadenylation would take place, after an A-rich region of 11 residues, and $154 \mathrm{bp}$ upstream of a $\mathrm{C}$-rich polypyrimidine tract of 14 nucleotides in length and $\mathrm{U} / \mathrm{C}$ ratio of 0.08 (5'CCCCC CCCCTCCCC $3^{\prime}$ ). It is a common feature that the presence of adenosine residue precedes the polyadenylation sites of a large number of trypanosome mRNAs [37]. It is believed that poly $(\mathrm{A})$ polymerases prefer an initial adenosine residue for attachment of the poly(A) tail, and the selection of the polyadenylation site would be strengthened by the presence of adenosine residues [38].

After determining the extent of the UTRs, it was possible to define the intergenic region (IR) within the $L$. 


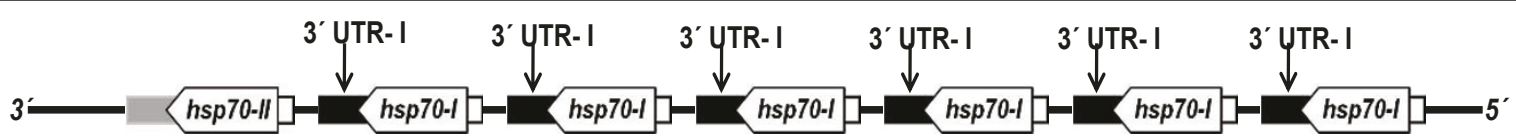

Figure 3 Graphical representation of the $3^{\prime}$ UTR-I position in the HSP70 cluster of L. braziliensis.

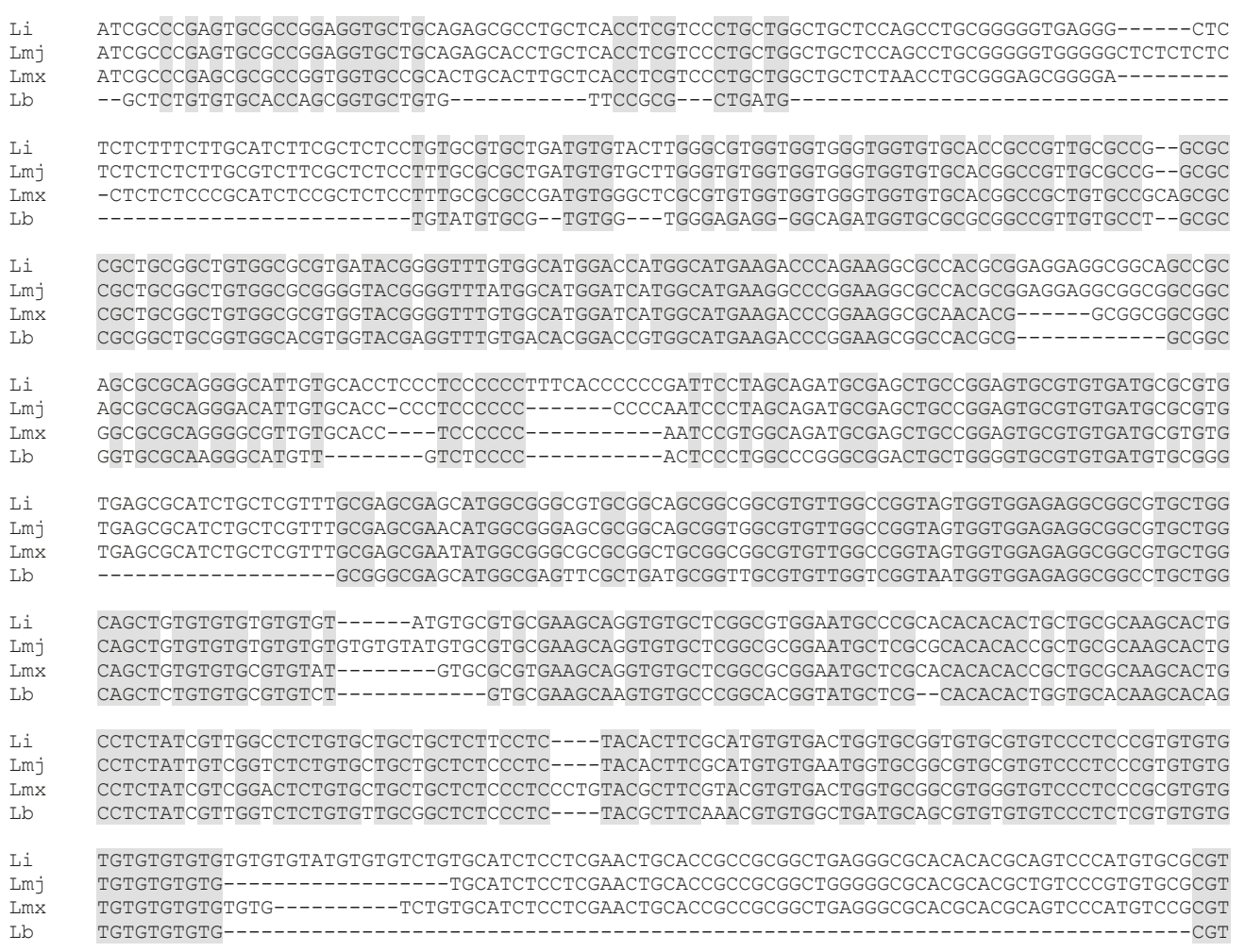

Li TGAGCGAGAGCGCTTGAGGCCGACCCTCTTTCCTCCCCCTACCCGTGTTCCGCGCTCCGTGTCCTACGACACCGACGAGGACCGCGCACA Lmj TGAGCGAGAGCGCTTGAGACCGACCCTCTTTCCTCCCCCTACCCGTGCTCCGCGCTCCGTGTCTTACGACACGGACCAGGACCGCGCATG Imx TGAGCGAGAGCGCTTGAGACCGACCCTCTTTCCTCCCCCTACCCGTGTTCCGCCCTCCGTGTCCTGCGGCACGGACGAGGACCGCGCACA Ib TGAGCGAGAGCGCATGAGACCGACTCTCTTTCCTCCCCTACCCGTACTCTGCGCTCCGTGTTCAGTGACACCGGCGAGGGCCGTGCATA

Li CGGCCAGCACTCACCCTGACTGCCCGCCGCG----CCTTTCTCTCCCTTGATGGAGTCCCTGTTAACCTTTACCGTCGCGCACGCACATG Imj GGGCCAGCGCTCACCCCGACTGCCCGCCGCG----CCTCTCTCTCCCTTGATGGAGCTCCTGTTAACCTTTACCGTCGCGCACACACATG Lmx TGGACAGCACTCACCCTGACTGCCCGCCGCG----CCT--CTCTCCCTTGATGGAGCCCCTGTTAACCTTTGCCGTCGCGCACACGCATG CGGCCAGCACTCACCCTGAATGCGCGCTGCGTCCCCCTCTCCCTCCCTCAGCTGAAGCCCTATTAACTTTTACCGGCACGCAC-------

Li CCGATGCCGCACCATCAGCGTACGGCATCCCCCTTCGCTTTTGTGCGTGCGCGTGTGCGCTTCTCCTT-TGTCTCTTCTCCTCCTTATTT Imj CCGATGCCGCACCATCAGCGTACGGCATCCCCCTTCGTTTTTGTGTGTGCGCGTGTGTGCTTCTCCTT-TGCCTCTTCACCTCCTTATTT Imx CCGATGCCGCGCCACCAGCGTACGGCATCCCCCTTCGCTTGTGTGCGTGCGCGTGTGCGCTTCGCCGT-TGTCTCTT---CTTCTTATTI Lb -CGAG--AGGGCACACGGCGTCC--CCCCCCCCCCCGCTT----GCGTGTGCGTGTGC-CCTCTCGGTGCCTCTCTT-TTCTTCTTTCCT

Li GCGGCAACTGTGCAGAGCGGGGGAGCGTCGCCGCCGGTGAGTCAGAGGGGAGACGGGG-AGGGAGACAGCGATGGAAGCACG-CCCCTCC Lmj GCGGCAACTCTGCAGAGCGGGGGAGCGCCGCTGCCGGTGACTCAAAGGGGAAACGGGGGAGGGAGACAGCGATGGAAGCACGCCCCCTCC

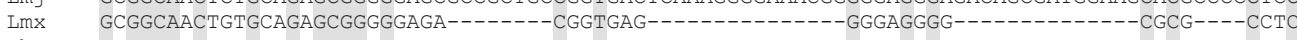
Lb GTGGCAGCG--GATGCGCGGAGCGACTCCTCTACCTCTCGCCATCTTTCGCCCCACGTCCGCGTG-----------CGCGCTCCCCCTCC

Li CCTCGCTGTCTCCCCCCCCCTATGCCCCCTCTTCGCCTAT-CATTTCAGTCTTGAGTTTCATCGATAAGAAGGCCCGACTCCGCGAGTGC Lmj CCTCGCTGTCTCCTCCCCCCT---CCCCCTCTTCGCCTAT-CATTTCAATCTTGAGTTTCATCGATAAGAAGGCCCGACTCCGCGAGTGC Lmx CATCGCTG--TCCTCCCCCCT--CCCTCCTCTTCGCCTAT-CTTTTCAGTCTTGAGCTTCCTCGATAGGAAGGCCCGACTCCGCGAGTGC $\mathrm{Lb}$ TGCCGCCTG-TCTTCCTCCCCTTCCCTCCTCCTCTGTTCCGTATTTCATTCCGGTGGTACATCGACAAGAAGG-ACGGCTCCGTGAGTGC

Li CGACCTGTGCCCCCTCCCCCTT-CCTTAA 1172

Lmj CGGCCTGTGCCCCCTCCCCCTTTCCTTAA 1158

$\begin{array}{lll}\text { Lmj } & \text { CGGCCTGTGCCCCCTCCCCCTTTCCTTAA } & 1158 \\ \text { Lmx } & \text { CGGCCTGTGCCCCCTCCCCCCT-CCTCAA } & 1093\end{array}$

Lb $\quad$ CGGCCTGTGCCCCTTCCCCTT-CCTTAA 936

Figure 4 Multiple sequence alignment among HSP70 3' UTR-I from L. braziliensis (Lb: JF449364), L. major (Lmj: LmjF28.2780), L. infantum (Li: LinJ28_V3.2960) and L. mexicana (Lmx). Conserved sequences are shaded in gray. L. major 3' UTR-I sequence was deduced by comparison with L. infantum and L. braziliensis $3^{\prime}$ UTR-I sequences. Additional file 3 , shows the sequence information, currently available in the GeneDB database, that was used for assembling of the putative L. mexicana 3' UTR-I sequence. 
braziliensis HSP70 locus. The IR, not included in the mature mRNA, is defined as the sequence beginning downstream of the polyadenylation site and ending immediately upstream of the spliced leader acceptor site of a downstream gene. Thus, the IR between LbrM28.2990 and LbrM28.2980 genes was found to be 237 nucleotides in length; this region was almost identical (99.6\%) to that cloned for this work (pLbHSP70-IR-E clone, GenBank accession number JF449366), suggesting a high degree of conservation of this region along the HSP70 cluster.

\section{Comparison of L. braziliensis HSP70 UTRs with their homologues in other Leishmania species}

The characterization of UTRs for L. braziliensis HSP70 genes (Figures 2, 3 and 4) has evidenced the existence of a remarkable conservation of the HSP7O locus along the genus Leishmania, extending previous studies [16] to a species of the Viannia subgenus. The 5' UTR cloned in this work was found to be highly conserved with the equivalent regions of the LbrM28_v2.2990 and LbrM28_v2.2980 genes (98\% and 99.5\%, respectively). The comparison with the HSP70 5' UTR of other Leishmania species showed also a remarkable sequence identity (77-81\%). Noticeably, this region was well conserved among all Leishmania species analyzed except for two exclusive sequences of L. braziliensis (Figure 2).

Comparison of the 3' UTR-I from L. braziliensis with those from other Leishmania species revealed identities between $71-73 \%$. Furthermore, stretches of identical nucleotides are present in the 3' UTR-I in all the species analyzed (Figure 3 and 4). Again, it was found that $L$. braziliensis sequence is the most divergent among the analyzed sequences. Thus, the L. braziliensis 3' UTR-I lacks several regions common to the other Leishmania species; in particular, there are two important sequence gaps, of 60 and $77 \mathrm{nts}$, located at the beginning and the middle of the region, respectively (Figure 3 and 4).

In contrast to the 3' UTR-I, the 3' UTR-II from $L$. braziliensis showed to be more divergent, having only a $55-57 \%$ of identity with the other species analyzed. Indeed, some sequences at the first half of the region were exclusive for L. braziliensis (Figure 5 and 6). Conversely, it was found that L. braziliensis sequence lacks several regions common to the other Leishmania species, especially in the second half of the region (Figure 5 and 6). Nevertheless, the presence of several short stretches of identical sequences among all the species analyzed was also found (Figure 5 and 6), suggesting a common evolutionarily origin.

\section{Expression analysis of HSP7O genes in L. braziliensis}

The identification of two divergent 3' UTR sequences for HSP70 genes in L. braziliensis and their comparison with the sequences in the GeneDB database allowed us to define the existence of two types of HSP7O genes, a question that remained to be solved [16]. Northern blot assays using different probes were performed in order to elucidate the expression of these genes in L. braziliensis. Using the IR-HSP70 probe, containing the complete 3'UTR-I together with 5'-UTR and short fragment of the coding region, two hybridization RNA species of very similar size were observed (Figure 7). In addition, a slight hybridizing fragment of about $5.6 \mathrm{~kb}$ was observed in all lanes, but its signal became stronger when parasites were incubated at $37^{\circ} \mathrm{C}$; accumulation of additional high molecular RNA species was also observed with this treatment (Figure 7A). It is likely that those fragments are representing RNA precursors (pre-mRNA), whose processing is disturbed by heat shock. The use of probes specific for 3' UTR-I and UTR-II allowed us to show the existence of both types of transcripts: the HSP70-II mRNA, which corresponds to the upper RNA in figure $7 \mathrm{~A}$, and the HSP70-I mRNA, which corresponds to the lower one. Of special interest, both types of transcripts did not show accumulation under heat shock treatment (Figure 7).

\section{Copy number and chromosomal location of HSP70 genes} in L. braziliensis

In order to estimate the number of HSP70 genes in the L. braziliensis locus, a Southern blot analysis was carried out (Figure 8). L. braziliensis total DNA was either totally or partially digested with selected restriction enzymes, and, after electrophoretic separation and transferring, hybridized with specific probes. A prominent 3.3-kb fragment was observed after digesting DNAs with either SmaI- or BamHI and hybridizing with the intercoding probe (H70-IR-E; Figure 8A), indicating that indeed the HSP7O locus in L. braziliensis should consist of tandemly repeated genes with a repetition unit of 3.3-kb. Additional fragments, showing lower hybridizations signals, were interpreted as representing the boundaries of the locus (Figure 8A,

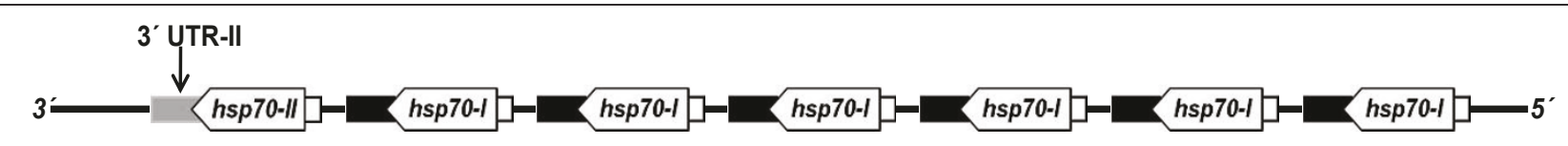

Figure 5 Graphical representation of the 3' UTR-II position in the HSP70 cluster of L. braziliensis. 


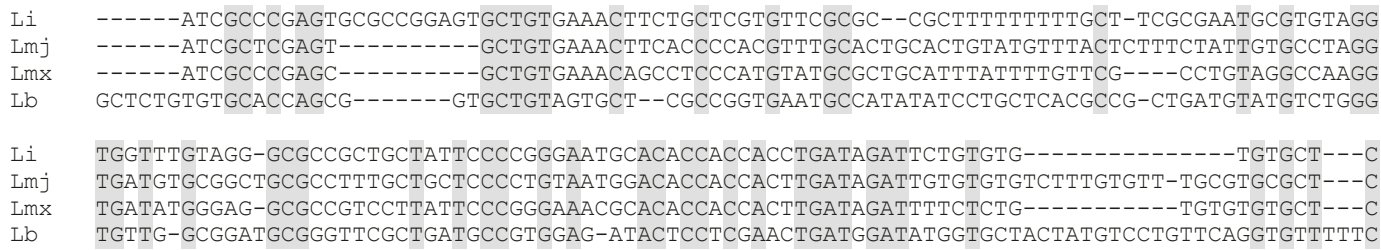

Li TCTGTAGCTCTGCTGCGC-----TTTTTCGAGTGC----CGTCGCTC------CGCGTGTCGTTTG-TGTCTAT-----TCTGAATTTGT Li TCTGTAGCTCTGCTGCGC-----TTTTTCGAGTGC----CGTCGCTC------CGCGTGTCGTTTG-TGTCTAT-----TCTGAATTTGT
Lmj TCCGTCGCTCTGCCGCAC-----TGTGTCGAGTGT----CATGAGTG------CGCGTGTTGTTCGGTGTCTAT-----TGTGAATTA-T Lmx TCCGTCCCGCTGCCGCGC-----TCTCTCGACTGC----CGTCGTTC------TGCGTGTCGTGTG-TGTCTCG-----TCTGAAGTATC Lb TGCCTTGCGTTGACGCGCGGGTGTCTTACAGTGGCGCTGCGACGCTCTCTCCTCGCATATGCGGCG-TGTCTCTGCGTGTGTGTATGTCT

Li TCTACGTTTTT-------TTTCCGTTTTATTTCGGAAGACTTCCGCGTGCTAAATCC---CCCCTTTCCCCCGTAACC-CCCCTCCCCC Lmj TCTGCATTTTTGGTT-TGTTTTGAATCTTATTTCGGAAGACTTCCGCGCGCTAAATCCTCCCCCCCCCCCCCCATTCCC-CCCTTCCCCC Lmx TCTGTTTTTTTTCG-----TTTGGG--TTGTTTCAAAAGACCCCCGCGCGTTAATATCCTCCTCCCCCCAACACACACT-CTTCTCCCCC

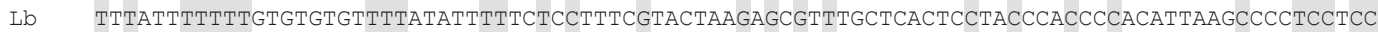

Li TTTTTTCTCTACG------tCTGTTTCGCCTTATTT---------------TATATATATATATAT---ATTATGGTACCGCCACC Lmj CTCCCCGCCCCCCGATAACTCTCCCTTCCCCCTTGTTTGTCTGCGTCCGTTTCGCTATATATATATATATGATATTATGGTATCGCCACG Lb CCTCTCCCCCTCCCG-------CATTTTGTTTTGTCT-------------TCAAAAATTAGTTCTCTCTTTGTT-TCCCGCCCTC

Li GCTCACGCTCTCGCATTCACA----GAGAGCCGTCCCTATCCACTCTTTCTCCCTCTTGTCCCTCACGCTGTCCATCGTACTAGCCACCC Lmj GCGCATGCTCTCGCATTCACA----GAGAGCCGTCTTTTTCCCCTCTCCCTCCCTCTTGTCCCTCAAGCTGTCCCTCGTACTACCCAGTC Lmx GCTCACCCTCTCGCGCACACACAGAGAGAGCCGTCCTTCTCCCCTCTCCCTCCCTCTTCTCCCTCACGCAGCCCCTCGTACTATTCACCC $\mathrm{Lb}$ TCTCGGTGTCTTGCG----CA----TGAACTCGCAGAGAGTCATCGCACACTCTCCTCTTCCTTAGTCTCTCCCTCTCTCTTTAACGCC

Li CCTCCCGC--CCCTACTTTTCCGCTGTCGGTCTTTCTAGTCCACACTCAAATACCACAACCACGGTGCCTGCGGCGCTGTGGGTGTGCGT Lmj CCTCCCGG--CCCCACTTTTCCGCTGTCGGTCTTTCCAGTCCACACCCAACTACCACGGCCACAGTGCGTGCGGCACGTTGTGTGTGCGT Lmx CCCCCCACGTCCCCACTCTTGTGCTGTCGGTCTTTCTCGTCCACACCCAACTACCACAGCTACGGCGCGTGCAGCACTGTG--TCTACGT Lb CCAACCTG--------------TTGTCGTTCTGTCTT--CCACACCCCACTACTGCGGTCACGCTGCATGTG------------TGTGT

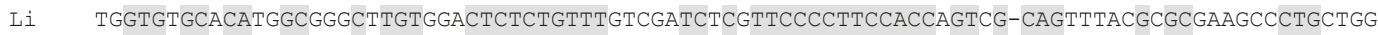
Lmj TGgTGtgCACATTGCAGgCTTGTAGACTCTCTGTTTGTCGTTCTCGTTCCCCTTCCACCAGTCG-CAGTTGAGGCGCTAAGCCCTGCTGG Lmx TGGTGTGCACATTGCAGGCGTGTTGACTCTCTGTTTGTTATTCTCGTTCCCCTTCCACCAGTCG-CAGGATCCGTGCAACGCTCTGCTGA Lb GTGTGGGCGCAGTGCCTTCTTGTGTTCTCTCTGTTT-----TCGCTTTCCCCTTCCACCCGTGGGCAGTAAAGGCGCATAAGCCTGTGCG

Li CTGGTGCGCGAGGAAGAGAGGCCCACCAGCCAGCGTGTGTGCCACACTCTCTGGCAGGG-TTGCTTGCCGGCTTTGTAGCTGAGGACGT Lmj CTGGTGCGCGAGGGAGGAGAGGCCCACTAGCCTGCGTGTGTGCTACACTCTCTGGTACAG-CTGCTTGCCAGCTGAGTAGCAGAGGACAT Lmx CTGGTGCGCGAGGGAGGAGAGGCCAACGAGCCTGCGTGTGTGCTACACTCTCTGGTAAGGTTTGCCTGCCAGCTTTGTAGCAGAGGACAT Lb CTGGTGCGCGAGGGAGGAGAGGACAACGAGCCCGCCTGTCTGCTATAATCGCCGGTGAAG-TTGTTCGACACCTTCGCAGGAGAGGAAGT

Li GGACAGATCACACCGTTGGGGGCGTAGAGAGAGGGAGCGTGCAG--TGGGTGAAAGCTCGGCAGGCGGGCGACGGCAGCGAGGATGCTCA Lmj GAATAGATCGCACCGTGGGAGGGGTAGAGAAAGGAAGTGTGCAGAGTGGGTGAAAGCTCGGCAGGCGGGCGACGGCAGCGAG-------Lmx GGATAGATCGCAGTGGTGGAGGCGTAGAGA GAGGGAGTGTGCAGTGTAGGTGAAAGCTCGGCAGGCGGGTGACGGCAGCGAGGATGCTCA

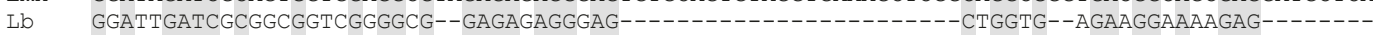

Li CTGCACGTCTGCATCGAgGTGCGTGTGGATGCACGCAAGCCGCCTCAGCA---ATCTACGCGTCTGTGTGTGTGCGAGAGAGCGAATtAA Lmj --_-----------GTGCACATGGATGCACGCAAGCTGGCTCAGCA---ATCTACGCGTCTGTGTGTGTGCGAGAGAGCGAATTAA Lmx CTGtAGGTCTGCATCGAGGTGCGTGTGGATGCACGCAAGCCGCCTCAGCAGCAATCTGCGCGTCTCTGTGTGTGCGAAAGGTCGAATCGA

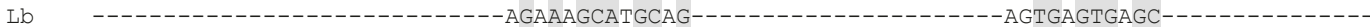

Li AGAAGAG---AAGAAGAGAGACGACGGAAGTGCGCGCGCAAACCAGGAAAGCCAACCGGCTTCCACACCCACAAACCCCCCG-TCGCTTT Lmj AgAAGAGAACATGAAGAGAGACGACGGAAGTGCGCGAGCAAACCCGGAAGGCCAACGGGCTTCCACACCCGCAAACCCCCG--TCGCGTC Lmx AGAAGAGAATAAGAAGAGAGACGACGGAAGTGCTCGAGCAAGCATG-AAAGCAAACGGGCTTCCACACCCACAAGCCCCCCTGTCGCGTC

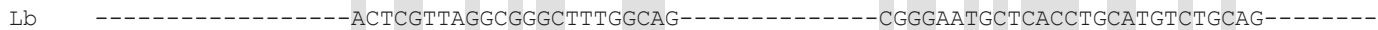

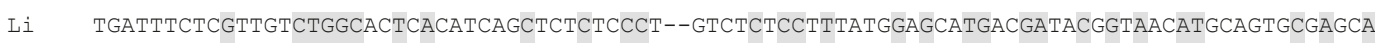
Lmj CGATCTCTCGTTTTCTGGCACTTACATGAGCTCTCTCCCTCTGTCTCTCCTTTATGGAGCATGTCGATACAGTAACATGCAGTGCGAGCA Lmx GAATCTCTCGTTGTCTGGCACTTAGGTCGGCTCCCTCCCTCTGTCTCTCCTTTGTGGAGCATGACGATACGGTAACATGCAGTGCAAGCA

Lb ------TGGAGGCTGGCGTTGATTCAGACAAGCCGCCAC-----CGCCATCTGTGCGTTTGTGGACGCGGGAGAAT------CAAAAA

Li CTGGAAAA 1071

Lmj CTGGAAAA 1096

LmX CTGGAAAA 1107

Lb AAAAAA-- 932

Figure 6 Multiple sequence alignment among HSP70 3'UTR-II from L. braziliensis (Lb: JF449365), L. major (Lmj: LmjF28.2770), L. infantum (Li: LinJ28_V3.3000) and L. mexicana (Lmx: LmxM.28.2770). Conserved sequences are shaded in gray. L. major and L. mexicana sequences were deduced by comparison with $L$. infantum and L. braziliensis $3^{\prime}$ UTR-II sequences. 


\section{A}
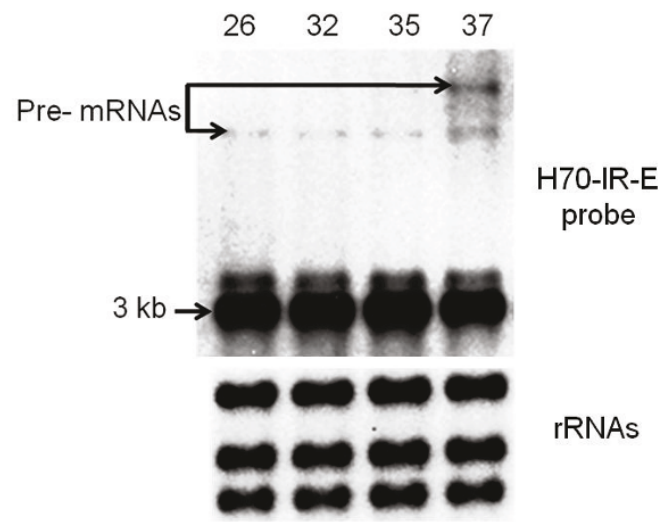

\section{B}

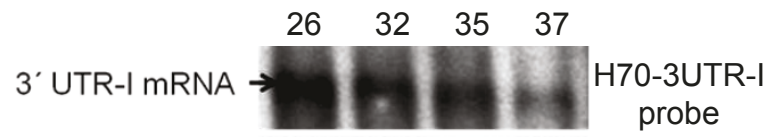
3. UTR-II mRNA $\rightarrow \quad \begin{gathered}\text { H70-3UTR-II } \\ \text { probe }\end{gathered}$

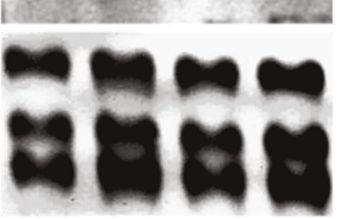

rRNAs

Figure 7 Identification of HSP7O-I and HSP70-II transcripts of L. braziliensis. Four micrograms of total RNA isolated from promastigotes incubated for $2 \mathrm{~h}$ at the indicated temperatures were separated on denaturant 1.5\% agarose/MOPS/formaldehyde gel, blotted and hybridized with the H70-IR-E (A), 3' UTR-I, and 3' UTR-II (B) probes. As load control, the agarose gels were stained with ethidium bromide (rRNA panels).
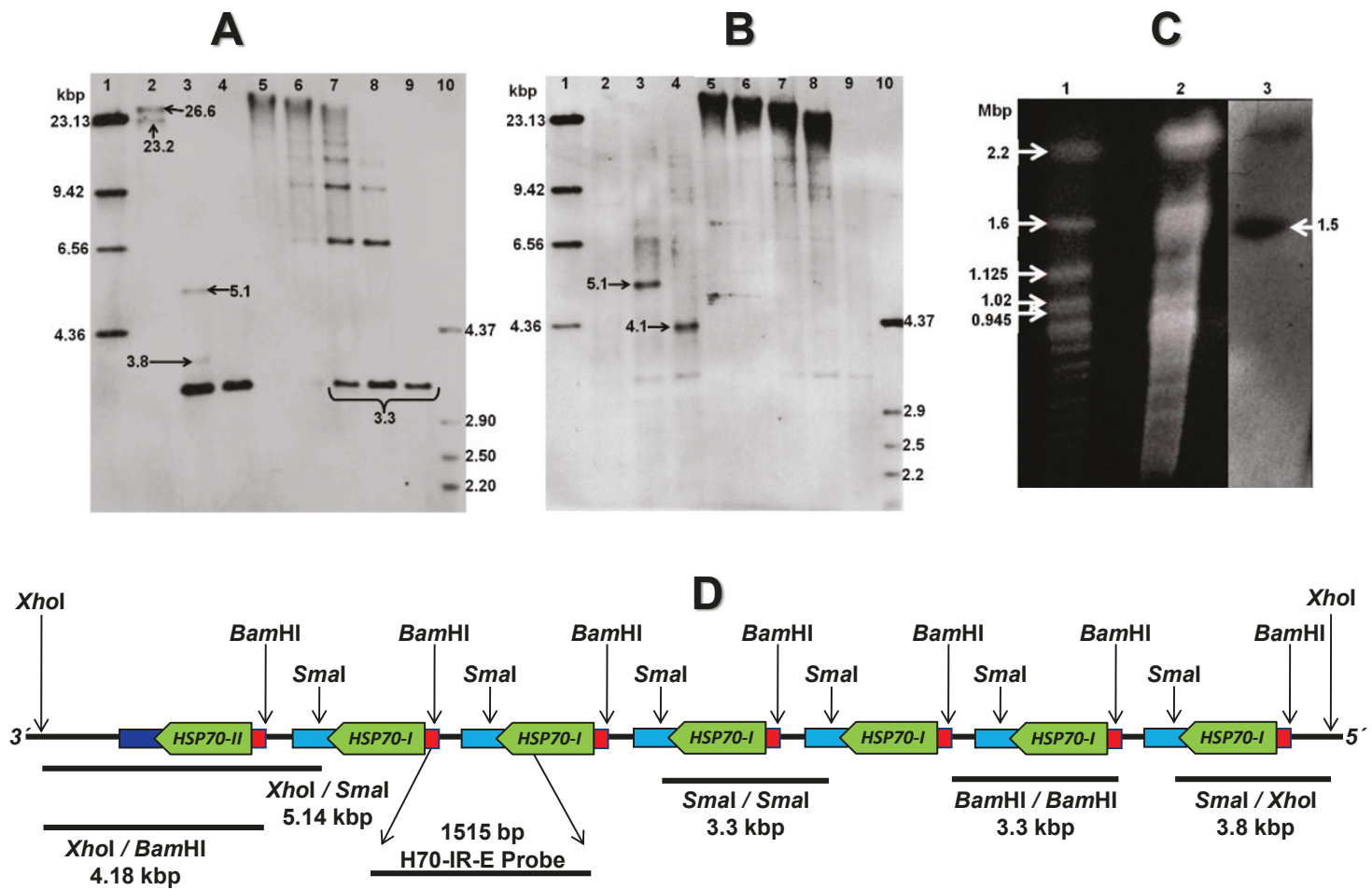

Figure 8 Genomic organization of HSP70 genes from L. braziliensis. DNA from promastigotes was totally or partially digested with restriction enzymes and the resulting fragments were separated on a $0.8 \%$ agarose gel, transferred to nylon membrane and hybridized with the H70-IR-E probe (A). The same blot was stripped and rehybridized with the HSP70-3' UTR-II probe (B). Lanes: 1, 185 ng of $\lambda$ DNA Hindlll; 2, 300 ng of Xholdigested DNA; 3, 1 g of Xhol+Smal-digested DNA; 4, 1 g of Xhol+BamHI-digested DNA; 5 to 9, DNA digested with BamHI for 2 min (lane 5), 5 min (lane 6), 15 min (lane 7), 30 min (lane 8), and 3 hours (lane 9); lane 10, 140 ng of $\Phi 29$ DNA+Hindlll. Molecular weight markers (lane 1 and 10) were labeled with digoxigenin and used as a probe in the hybridizations. (C) Pulsed field gel electrophoresis showing ethidium bromide staining of S. cerevisiae (lane 1), and L. braziliensis chromosomes (lane 2). Panel 3 shows the hybridization of the H70-IR-E probe to the L. braziliensis chromosomes. (D) Graphical representation of the deduced physical map for HSP70 locus in L. braziliensis. Small red boxes represent the 5' UTR; light blue boxes the 3' UTR-I, dark blue one the 3' UTR-II, and green boxes the ORFs. 
lane 3 , and $8 \mathrm{~B}$, lanes 3 , and 4 ). The tandem organization of the locus was demonstrated by BamHI partial digestion of L. braziliensis DNA; a typical ladder was observed (Figure 8A, lanes 5 to 8 ).

Hybridization of the membrane with the 3' UTR-II probe showed a sole hybridization fragment $(5.1-\mathrm{kb}$ in XhoI+SmaI digested DNA, and 4.1-kb in XhoI+BamHI digested DNA) (Figure 8B, lanes 3 and 4, respectively), in agreement with the existence of a unique HSP70-II gene, which was located at the 3' end of the locus (Figure $8 \mathrm{D})$. According to the number of fragments observed in the lanes containing BamHI partially digested DNA (Figure 8A, lanes 5 to 8), and taking into account the size of the hybridizing XhoI-fragments which should contain the complete locus (Figure 8A, lane 2), it was estimated the presence of around seven HSP7O genes in the L. braziliensis HSP7O locus, as is shown in Figure 8D which summarizes the genomic organization of the L. braziliensis HSP70 locus.

The presence of two hybridization fragments, larger than $20 \mathrm{~kb}$, in the lane containing XhoI-digested DNA (Figure 8A, lane 2) has no direct explanation. Two hypotheses may be invoked to explain this unexpected finding. On the one hand, it can be postulated the existence of two identical HSP7O tandems that, furthermore, should be found in the same chromosome. Thus, PFGE separation and hybridization assays showed that HSP7O genes are located in a chromosome of approximately 1.5 $\mathrm{Mb}$ (Figure $8 \mathrm{C}$ ), which would correspond to the chromosome 28, according to the location of the HSP70 locus in the GeneDB database. The other hypothesis is the existence of allelic polymorphisms either within the locus (affecting the number of HSP70 gene copies) or in its boundaries (affecting one of the XhoI restriction sites). Although the size and hybridization intensity of these $X h o I$ restriction fragments supports the second alternative, and the resolution of the PFGE assay do not permit distinguish between the two size different sister chromosomes, further work is needed to clarify this finding.

\section{Conclusions}

The present work was intended to establish the genomic organization of HSP70 genes in L. braziliensis. Firstly, by RT-PCR and cloning, we identified two types of 3' UTR sequences, demonstrating that also in L. braziliensis two types of HSP70 genes exist, a feature shared with other Leishmania species. In addition, our analyses support the existence of at least seven HSP7O genes arranged in a head-to-tail manner. In summary, the HSP70 locus in L. braziliensis, like in Leishmania subgenus species, is composed of the two types of genes (HSP70-I and HSP70-II), the number and the relative position of these genes being very similar in the Leishmania genus. This finding is of especial value taking into account that $L$. $(V$.) braziliensis complex is considered as the earliest divergent species of the genus Leishmania [39]. The strict conservation of the HSP70 gene array in all Leishmania species analyzed suggests that this type of arrangement must have an important functional role. Indeed, as recently reported, the HSP70-II gene in L. infantum is extremely relevant for virulence and intracellular survival of the parasite [40].

Additionally, we have experimentally mapped the 5' and 3' UTR of both types of HSP70 genes of L. braziliensis. After comparing with the genomic sequences, the position of processing signals, such as the trans-splicing and polyadenylation sites as well as the C-rich polypyrimidine tracts, were determined. The distance between these elements is in agreement with previously reported range of distances [41].

Former studies in L. braziliensis reported that after probing the mRNA blot with the HSP70 coding region, two hybridization RNA species very close in size were observed, corresponding the lower molecular weight species to that hybridizing with a 3' UTR-I probe [16]. Our Northern blot analysis supported these findings and revealed that the larger RNA corresponds to the HSP70II transcript, confirming the existence of the HSP70-II genes in this parasite. Although, the size difference between these transcripts could not be explained by the sole difference in size of both 3' UTRs, it is likely that the differences are due to different length of the poly(A) tail $[42,43]$. Noticeably, it is considered that unstable mRNAs carry shorter poly (A) tails $[44,45]$. Northern blot assays showed that the steady-state levels of both transcripts are not affected by the temperature of incubation. Consequently, in contrast to the species of the Leishmania subgenus, L. braziliensis HSP70-I transcripts are not stabilized upon heat shock.

\section{Additional material}

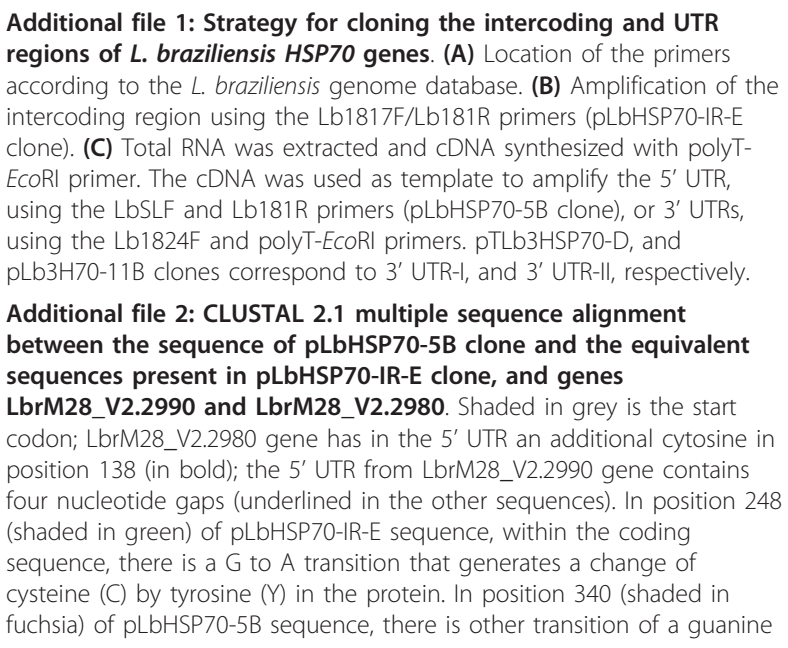


for an adenine that generates a change of Glutamic acid (E) by Lysine (K).

Additional file 3: Location of HSP70 genes in the L. mexicana genome. The upper black arrows demarcate the HSP70 locus. Red boxes indicate the 5' UTR, blue arrows the 3' UTR-I, green arrow the 3' UTR-II, and red stars the gaps in the sequence. L. mexicana $3^{\prime}$ UTR-I sequence was assembled from three fragments (GeneDB positions: 1'048.970 1'049.137; 1'047.042 - 1'047.656, and 1'045.959 - 1'046.951) deduced by comparison of the L. mexicana genome with the GenBank entry

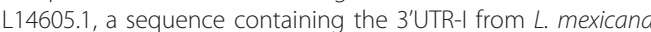
amazonensis. L. mexicana 3' UTR-II sequence was deduced by comparison with $L$. infantum and $L$. braziliensis $3^{\prime}$ UTR-II sequences.

\section{Abbreviations}

CL: cutaneous leishmaniasis; MCL: muco-cutaneos leishmaniasis; HSP: heat shock protein; HSP70: 70 kDa heat shock protein; IR: Intergenic region; PFGE: pulsed field gel electrophoresis; PPT: thermosensitive polypyrimidine-rich element; UTR: untranslated region; VL: visceral leishmaniasis; LACK: activated protein kinase $\mathrm{c}$ receptor (guanine nucleotide-binding protein beta subunitlike protein).

\section{Acknowledgements}

CJP's lab was supported by Colciencias (Colombia), Research project No. 1203-405-20233. JMR's lab was supported by grants from the Ministerio de Ciencia y Tecnología (BFU2009-08986) and the Fondo de Investigaciones Sanitarias (ISCIII-RETIC RD06/0021/0008-FEDER). CAR was supported by Colciencias, Programa Nacional de Doctorados, convocatoria 2008.

\section{Author details}

'Laboratorio de Parasitología Molecular, Departamento de Microbiología, Facultad de Ciencias, Pontificia Universidad Javeriana, Carrera 7 No. 43-82, Edificio 52, Oficina 608, Bogotá, Colombia. ${ }^{2}$ Centro de Biología Molecular "Severo Ochoa" (CSIC-UAM), Universidad Autónoma de Madrid, 28049 Madrid, Spain.

\section{Authors' contributions}

CAR, JMR, and CJP conceived and designed the experiments. CAR performed the experiments. CAR, JMR, and CJP analyzed the data. CAR, JMR, and CJP wrote the paper. All authors revised and approved the final version of the manuscript.

\section{Competing interests}

The authors declare that they have no competing interests.

Received: 12 July 2011 Accepted: 26 August 2011

Published: 26 August 2011

\section{References}

1. Murray HW, Berman JD, Davies CR, Saravia NG: Advances in leishmaniasis. Lancet 2005, 366(9496):1561-1577.

2. Control of the leishmaniases. [http://whqlibdoc.who.int/trs/ WHO_TRS_949_eng.pdf].

3. Oddone R, Schweynoch C, Schonian G, de Sousa Cdos S, Cupolillo E, Espinosa D, Arevalo J, Noyes H, Mauricio I, Kuhls K: Development of a multilocus microsatellite typing approach for discriminating strains of Leishmania (Viannia) species. J Clin Microbiol 2009, 47(9):2818-2825.

4. Dantas-Torres F: Canine leishmaniosis in South America. Parasit Vectors 2009, 2(Suppl 1):S1.

5. Shapira M, McEwen JG, Jaffe CL: Temperature effects on molecular processes which lead to stage differentiation in Leishmania. EMBO J 1988, 7(9):2895-2901.

6. Maresca B, Kobayashi GS: Hsp70 in parasites: as an inducible protective protein and as an antigen. Experientia 1994, 50(11-12):1067-1074.

7. Quijada L, Soto M, Alonso C, Requena JM: Analysis of post-transcriptional regulation operating on transcription products of the tandemly linked Leishmania infantum hsp70 genes. J Biol Chem 1997, 272(7):4493-4499.

8. Krobitsch S, Clos J: A novel role for $100 \mathrm{kD}$ heat shock proteins in the parasite Leishmania donovani. Cell Stress Chaperones 1999, 4(3):191-198.
9. Zilka A, Garlapati S, Dahan E, Yaolsky V, Shapira M: Developmental regulation of heat shock protein 83 in Leishmania. $3^{\prime}$ processing and mRNA stability control transcript abundance, and translation id directed by a determinant in the $3^{\prime}$-untranslated region. J Biol Chem 2001, 276(51):47922-47929.

10. Bente M, Harder S, Wiesgigl M, Heukeshoven J, Gelhaus C, Krause E, Clos J, Bruchhaus I: Developmentally induced changes of the proteome in the protozoan parasite Leishmania donovani. Proteomics 2003, 3(9):1811-1829.

11. Hartl FU: Molecular chaperones in cellular protein folding. Nature 1996, 381(6583):571-579

12. Bukau B, Horwich AL: The Hsp70 and Hsp60 chaperone machines. Cell 1998, 92(3):351-366.

13. Mayer MP, Bukau B: Hsp70 chaperones: cellular functions and molecular mechanism. Cell Mol Life Sci 2005, 62(6):670-684.

14. Folgueira C, Requena JM: A postgenomic view of the heat shock proteins in kinetoplastids. FEMS Microbiol Rev 2007, 31(4):359-377.

15. Folgueira C, Quijada L, Soto M, Abanades DR, Alonso C, Requena JM: The translational efficiencies of the two Leishmania infantum HSP70 mRNAs, differing in their 3 '-untranslated regions, are affected by shifts in the temperature of growth through different mechanisms. J Biol Chem 2005, 280(42):35172-35183

16. Folgueira C, Canavate C, Chicharro C, Requena JM: Genomic organization and expression of the HSP70 locus in New and Old World Leishmania species. Parasitology 2007, 134(Pt 3):369-377.

17. Requena JM: Lights and shadows on gene organization and regulation of gene expression in Leishmania. Front Biosci 2011, 17:2069-2085.

18. Charest $\mathrm{H}$, Zhang WW, Matlashewski $\mathrm{G}$ : The developmental expression of Leishmania donovani $\mathrm{A} 2$ amastigote-specific genes is posttranscriptionally mediated and involves elements located in the 3'untranslated region. J Biol Chem 1996, 271(29):17081-17090.

19. Mishra KK, Holzer TR, Moore LL, LeBowitz JH: A negative regulatory element controls mRNA abundance of the Leishmania mexicana Paraflagellar rod gene PFR2. Eukaryot Cell 2003, 2(5):1009-1017.

20. Larreta R, Soto M, Quijada L, Folgueira C, Abanades DR, Alonso C, Requena JM: The expression of HSP83 genes in Leishmania infantum is affected by temperature and by stage-differentiation and is regulated at the levels of mRNA stability and translation. BMC Mol Biol 2004, 5:3.

21. Purdy JE, Donelson JE, Wilson ME: Leishmania chagasi: the alpha-tubulin intercoding region results in constant levels of mRNA abundance despite protein synthesis inhibition and growth state. Exp Parasitol 2005, 110(2):102-107.

22. Zick A, Onn I, Bezalel R, Margalit H, Shlomai J: Assigning functions to genes: identification of S-phase expressed genes in Leishmania major based on post-transcriptional control elements. Nucleic Acids Res 2005 33(13):4235-4242.

23. Holzer TR, Mishra KK, LeBowitz JH, Forney JD: Coordinate regulation of a family of promastigote-enriched mRNAs by the $3^{\prime} U T R$ PRE element in Leishmania mexicana. Mol Biochem Parasitol 2008, 157(1):54-64.

24. David M, Gabdank I, Ben-David M, Zilka A, Orr I, Barash D, Shapira M: Preferential translation of Hsp83 in Leishmania requires a thermosensitive polypyrimidine-rich element in the $3^{\prime}$ UTR and involves scanning of the 5' UTR. RNA 2010, 16(2):364-374.

25. Peacock CS, Seeger K, Harris D, Murphy L, Ruiz JC, Quail MA, Peters N, Adlem E, Tivey A, Aslett $M$, et al: Comparative genomic analysis of three Leishmania species that cause diverse human disease. Nat Genet 2007, 39(7):839-847.

26. Sambrook JRD: Molecular Cloning: A Laboratory Manual. New York; 2 2001

27. Quijada L, Soto M, Alonso C, Requena JM: Identification of a putative regulatory element in the $3^{\prime}$-untranslated region that controls expression of HSP70 in Leishmania infantum. Mol Biochem Parasitol 2000 110(1):79-91.

28. Lee MG, Atkinson BL, Giannini SH, Van der Ploeg LH: Structure and expression of the hsp 70 gene family of Leishmania major. Nucleic Acids Res 1988, 16(20):9567-9585.

29. Hausler T, Clayton C: Post-transcriptional control of hsp70 mRNA in Trypanosoma brucei. Mol Biochem Parasitol 1996, 76(1-2):57-71.

30. Lee MG: The $3^{\prime}$ untranslated region of the hsp 70 genes maintains the level of steady state mRNA in Trypanosoma brucei upon heat shock. Nucleic Acids Res 1998, 26(17):4025-4033. 
31. Engman DM, Sias SR, Gabe JD, Donelson JE, Dragon EA: Comparison of HSP70 genes from two strains of Trypanosoma cruzi. Mol Biochem Parasitol 1989, 37(2):285-287.

32. Requena JM, Lopez MC, Jimenez-Ruiz A, de la Torre JC, Alonso C: A headto-tail tandem organization of hsp70 genes in Trypanosoma cruzi. Nucleic Acids Res 1988, 16(4):1393-1406.

33. Requena JM, Lopez MC, Jimenez-Ruiz A, Morales G, Alonso C: Complete nucleotide sequence of the hsp70 gene of T. cruzi. Nucleic Acids Res 1989, 17(2):797.

34. Requena JM, Jimenez-Ruiz A, Soto M, Assiego R, Santaren JF, Lopez MC, Patarroyo ME, Alonso C: Regulation of hsp70 expression in Trypanosoma cruzi by temperature and growth phase. Mol Biochem Parasitol 1992, 53(1-2):201-211.

35. de Carvalho EF, de Castro FT, Rondinelli E, Soares CM, Carvalho JF: HSP 70 gene expression in Trypanosoma cruzi is regulated at different levels. J Cell Physiol 1990, 143(3):439-444.

36. Rodrigues DC, Silva R, Rondinelli E, Urmenyi TP: Trypanosoma cruzi: modulation of HSP70 mRNA stability by untranslated regions during heat shock. Exp Parasitol 2010, 126(2):245-253.

37. Matthews KR, Tschudi C, Ullu E: A common pyrimidine-rich motif governs trans-splicing and polyadenylation of tubulin polycistronic pre-mRNA in trypanosomes. Genes Dev 1994, 8(4):491-501.

38. Stiles JK, Hicock Pl, Shah PH, Meade JC: Genomic organization, transcription, splicing and gene regulation in Leishmania. Ann Trop Med Parasitol 1999, 93(8):781-807.

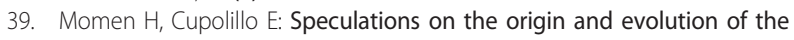
genus Leishmania. Mem Inst Oswaldo Cruz 2000, 95(4):583-588.

40. Carrion J, Folgueira C, Soto M, Fresno M, Requena JM: Leishmania infantum HSP70-II null mutant as candidate vaccine against leishmaniasis: a preliminary evaluation. Parasit Vectors 2011, 4(1):150

41. LeBowitz JH, Smith HQ, Rusche L, Beverley SM: Coupling of poly(A) site selection and trans-splicing in Leishmania. Genes Dev 1993, 7(6):996-1007.

42. Dewannieux $M$, Heidmann T: Role of poly(A) tail length in Alu retrotransposition. Genomics 2005, 86(3):378-381.

43. van Leeuwen HC, Liefhebber JM, Spaan WJ: Repair and polyadenylation of a naturally occurring hepatitis $C$ virus $3^{\prime}$ nontranslated region-shorter variant in selectable replicon cell lines. J Virol 2006, 80(9):4336-4343.

44. Shapira M, Zilka A, Garlapati S, Dahan E, Dahan I, Yavesky V: Post transcriptional control of gene expression in Leishmania. Med Microbiol Immunol 2001, 190(1-2):23-26.

45. Schwede A, Ellis L, Luther J, Carrington M, Stoecklin G, Clayton C: A role for Caf1 in mRNA deadenylation and decay in trypanosomes and human cells. Nucleic Acids Res 2008, 36(10):3374-3388.

doi:10.1186/1756-3305-4-166

Cite this article as: Ramírez et al.: Identification of the HSP70-II gene in Leishmania braziliensis HSP70 locus: genomic organization and UTRs characterization. Parasites \& Vectors 2011 4:166.

\section{Submit your next manuscript to BioMed Central and take full advantage of:}

- Convenient online submission

- Thorough peer review

- No space constraints or color figure charges

- Immediate publication on acceptance

- Inclusion in PubMed, CAS, Scopus and Google Scholar

- Research which is freely available for redistribution 